INT. J. PROD. RES., 2001, VOL. 39, NO. 13, 2991-3002

\title{
A new approach for evaluation of risk priorities of failure modes in FMEA
}

\author{
FIORENZO FRANCESCHINI $\dagger^{*}$ and MAURIZIO GALETTO $\dagger$
}

This paper presents a method for carrying out the calculus of the risk priority of failures in Failure Mode and Effect Analysis (FMEA). The novelty of the method consists of new management of data provided by the design team, normally given on qualitative scales, without necessitating an arbitrary and artificial numerical conversion. The practical effects of these issues are shown in an application example.

\section{Preliminary considerations}

Since its introduction as a support tool for designers, FMEA (Failure Mode and Effect Analysis) has been extensively used in a wide range of contexts (Stamatis 1995, Hatty and Owens 1995, Bowles 1998). The great number of papers published in various technological and service areas bear witness to this interest (Hatty and Owens 1995, Wirth et al. 1996).

Designers' interest in FMEA is due to its capacity to perceive two very important aspects:

- the capability of stimulating the application of the continuous improvement concept in design (Franceschini and Rossetto 1995b);

- the possibility of methodical documenting of the design evolution.

FMEA is a reliability tool, which requires identifying failure modes of a specific product or system, their frequency and potential causes. It is normally applied by an interfunctional work team, with the right know-how to analyse the whole product life cycle.

As a result of its application, it allows 'quantifying' how 'dangerous' a failure mode is, and also provides a rank of risk priorities of failure modes and a list of corrective actions to remove them.

A typical form used for FMEA development is illustrated in table 1. It shows a list of items that identify:

- the system or component part

- the potential failure mode

- the potential effect of failure

- the severity index $(S)$

Revision received March 2001.

$\dagger$ Politecnico di Torino, Dipartimento di Sistemi di Produzione ed Economia dell'Azienda, Corso Duca degli Abruzzi, 24 - 10129 Torino, Italy.

* To whom correspondenœ should be addressed. e-mail: fiorenzo@athena.polito.it 


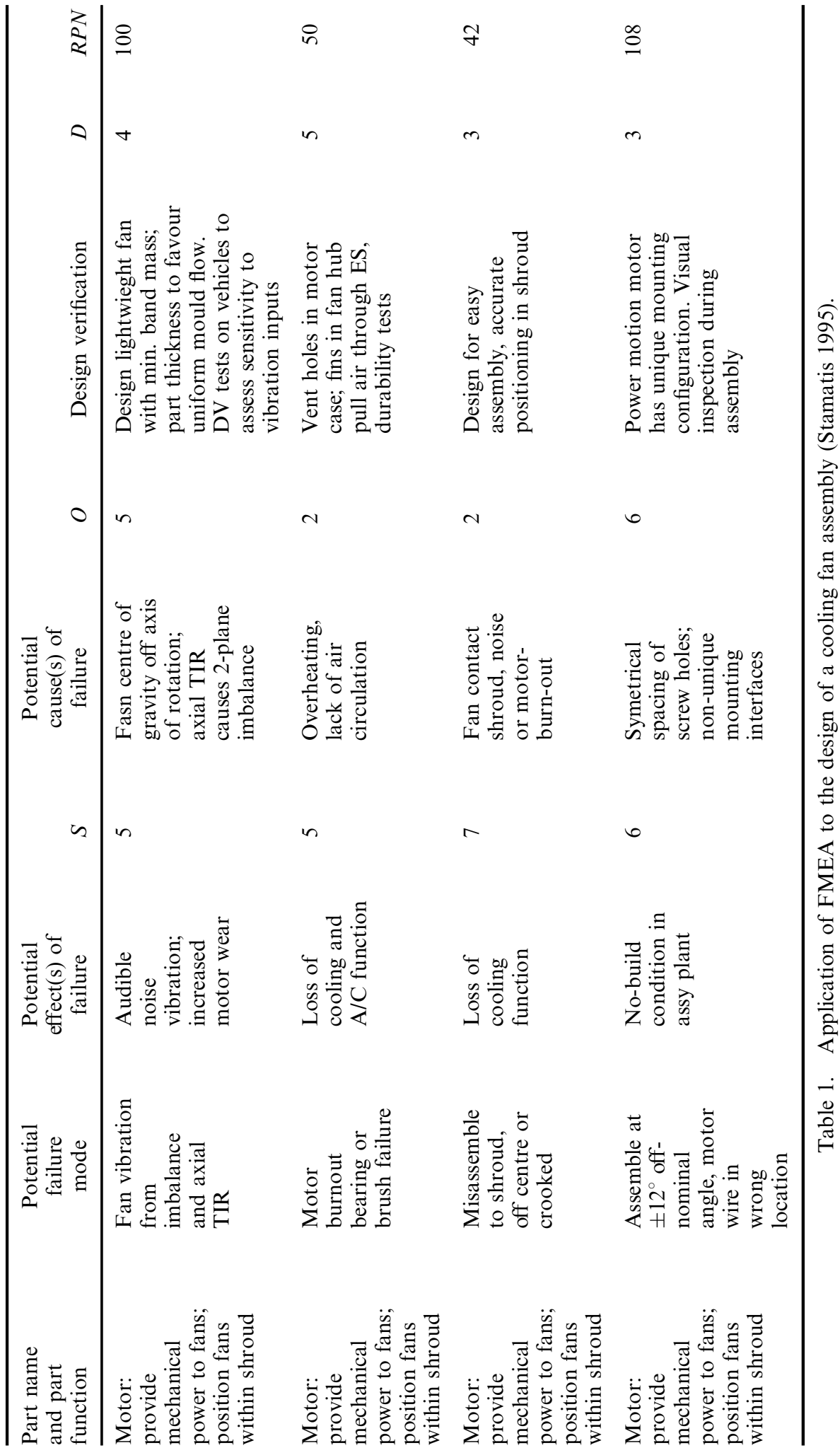


- the potential cause of failure

- the frequency of occurrence index $(O)$

- the design verification actions

- the detectability index $(D)$

- the Risk Priority Number $(R P N)$.

The characteristic failure mode indexes are expressed on ordinal qualitative scales (Fraser 1994, Franceschini and Rossetto 1997) identifying the various levels of 'dangerous' situations. Tables 2, 3 and 4 show the qualitative scales mostly used for the severity, the detectability and the occurrence indexes (Stamatis 1995). It is assumed that all index scales have the same number of scale levels.

For a generic design, after the identification of failure modes, effects and causes of a possible occurrence, the Risk Priority Number $(R P N)$ is calculated. $R P N$ is an index that expresses the risk level priority associated with each failure mode.

In the traditional FMEA approach, the $R P N$ index is determined by calculating the product of the three indexes: severity, frequency and detection:

$$
R P N=S \cdot O \cdot D
$$

In the $R P N$ calculation, the assigned values on the three index qualitative scales are interpreted as being numbers. 'Information initially gathered on the qualitative scales' is therefore arbitrarily interpreted and utilized on a quantitative scale with different properties from the first one.

\begin{tabular}{|c|c|c|}
\hline & Level & Criteria \\
\hline No & 1 & No effect. \\
\hline Very slight & 2 & $\begin{array}{l}\text { Customer not annoyed. Very slight effect on product or system } \\
\text { performance. }\end{array}$ \\
\hline Slight & 3 & $\begin{array}{l}\text { Customer slightly annoyed. Slight effect on product or system } \\
\text { performance. }\end{array}$ \\
\hline Minor & 4 & $\begin{array}{l}\text { Customer experiences minor nuisance. Minor effect on product or } \\
\text { system performance. }\end{array}$ \\
\hline Moderate & 5 & $\begin{array}{l}\text { Customer experiences some dissatisfaction. Moderate effect on } \\
\text { product or system performance. }\end{array}$ \\
\hline Significant & 6 & $\begin{array}{l}\text { Customer experiences discomfort. Product performance } \\
\text { degraded, but operable and safe. Partial failure, but operable. }\end{array}$ \\
\hline Major & 7 & $\begin{array}{l}\text { Customer dissatisfied. Product performance severely affected but } \\
\text { functionable and safe. System impaired. }\end{array}$ \\
\hline Extreme & 8 & $\begin{array}{l}\text { Customer very dissatisfied. Product inoperable but safe. System } \\
\text { inoperable. }\end{array}$ \\
\hline Serious & 9 & $\begin{array}{l}\text { Potential hazardous effect. Able to stop product without } \\
\text { mishap - time dependent failure. Compliance with government } \\
\text { regulation is in jeopardy. }\end{array}$ \\
\hline Hazardous & 10 & $\begin{array}{l}\text { Hazardous effect. Safety related-sudden failure. Non- } \\
\text { compliance with government regulation. }\end{array}$ \\
\hline
\end{tabular}

Table 2. Qualitative scale for the severity index (S) (Stamatis 1995). 


\begin{tabular}{lcl}
\hline Effect & Level & \multicolumn{1}{c}{ Criteria } \\
\hline Almost never & 1 & Failure unlikely. History shows no failure. \\
Remote & 2 & Rare number of failures likely. \\
Vert slight & 3 & Very few failures likely. \\
Slight & 4 & Few failures likely. \\
Low & 5 & Occasional number of failures likely. \\
Medium & 6 & Medium number of failures likely. \\
Moderately high & 7 & Moderately high number of failures likely. \\
High & 8 & High number of failures likely. \\
Very high & 9 & Very high number of failures likely. \\
Almost certain & 10 & Failure almost certain. History of failures exists from previous \\
& & or similar designs. \\
\hline
\end{tabular}

Table 3. Qualitative scale for the occurrence index $(O)$ (Stamatis 1995).

\begin{tabular}{lcl}
\hline Effect & Level & \multicolumn{1}{c}{ Criteria } \\
\hline Almost certain & 1 & Proven detection methods available in concept stage. \\
Very high & 2 & Proven computer analysis available in early design stage. \\
High & 3 & Simulation and/or modelling in early stage. \\
Moderately high & 4 & Tests on early prototype system elements. \\
Medium & 5 & Tests on preproduction system components. \\
Low & 6 & $\begin{array}{l}\text { Tests on similar system components. } \\
\text { Slight }\end{array}$ \\
Very slight & 7 & $\begin{array}{l}\text { Tests on product with prototypes with system components } \\
\text { installed. }\end{array}$ \\
Remote & 8 & $\begin{array}{l}\text { Proving durability tests on products with system components } \\
\text { installed. }\end{array}$ \\
Almost & 9 & Only unproven or unreliable technique(s) available. \\
imposible & 10 & No known techniques available. \\
\hline
\end{tabular}

Table 4. Qualitative scale for the detectability index (D) (Stamatis 1995).

In other words, the original ordinal scale is transformed in a new cardinal scale characterized by a metric and by the integer number composition properties.

The $R P N$ is thus defined on a rather special scale, which, moreover, does not completely cover the range $[1,1000]$ of the integers because there are, for example, some 'holes' corresponding to prime numbers contained in the range itself.

This arbitrary 'promotion' of the scale properties brings about a series of problems in the $R P N$ interpretation. In more detail, the data numbering involves:

- the definition of the $R P N$ on a formally wider scale than that of the three component indexes, which generates a fictitious increase of its resolution; 
- the assumption that the scales of the three $S, O$ and $D$ indexes have the same metric and that the same danger level corresponds to the same values on different index scales;

- the assumption that the three failure mode indexes are all equally important;

- the possibility of identifying, with the same $R P N$, situations characterized by different danger index levels. For example, the condition assigning to $(S, O, D)$ indexes the values $(8,1,1)$ is considered at the same level as $(2,2,2)$. Both situations determine an $R P N=8$. But is this statement legitimate?

The numeric data interpretation brings about the simplification of the $R P N$ calculation; however, it also increases the risk of moving its meaning away from the logic of the design team that supplied the figures.

The numbering - acknowledging 'metrological properties' higher than actually possessed by collected information - can therefore cause a 'distortion' effect, which can partially or completely distort the contents (Franceschini and Rossetto 1995a, 1998).

Other methods have been proposed for the $R P N$ calculation in the literature (Bowles and Pelaez 1995, Goossens and Cooke 1997). However, they do not remove some of the complexities illustrated during the discussion. In particular, these methods are quite complex to manage and require the definition of special functions and/or a know-how that is not always available to designers. These issues stimulated the idea of setting up an alternative method to the traditional one. This method is able to solve some of the questions raised and, in particular, the need to introduce non-existing properties to estimate the $R P N$ index. It also allows the design team to implement flexible strategies to detect the most dangerous failure modes.

The method also provides the possibility of considering the difference in importance of the characteristic indexes, so avoiding a further work burden for designers.

At the end of the discussion, an example of the new approach together with a comparison with the traditional procedure will be provided.

\section{The method}

The main aim of defining failure mode priorities is to draw the designer's attention towards the most dangerous failure modes for the product. For this to be an important effort improving the design quality, it must not alter the content of the information supplied by the design team during the analysis.

The proposed method is able to deal with information expressed on an ordered qualitative scale with no need to resort to an artificial numerical conversion of the scale. It can be classified within the class of ME-MCDM techniques (Multi Expert — Multiple Criteria Decision Making) (Yager 1993).

The use of qualitative scales raises a few issues for data processing. For example, in using numeric scales, the difference operation between two scale elements is defined, but this does not happen for qualitative scales, which have ordinal properties only.

The method is inspired by the work of Bellman and Zadeh, lately 'enriched' by Yager, for the solution of multi-criteria decision-making problems (Bellman and Zadeh 1970, 1975, 1976, Yager 1981, Yager and Filev 1994). In fact, FMEA can be considered as a decision-making support tool for designers. The decision consists 
of defining the order to analyse (from a design point of view) the failure mode effects of the considered product.

Characteristic indexes can be interpreted as evaluation criteria $g_{j}$ (with $j=1, \ldots, n$ ), while failure modes as the alternatives $a_{i}$ (with $i=1, \ldots, m$ ) to be selected.

The method considers each decision-making criterion (characteristic index) as a 'fuzzy' subset over the set of alternatives to be selected.

The grade of membership of alternative $a_{i}$ in $g_{j}$ indicates the degree to which $a_{i}$ satisfies the criterion specified.

The model suggests a two-step procedure.

(i) Aggregation of evaluations expressed on each criterion for a given alternative $\left(a_{i}\right)$

$$
R P C\left(a_{i}\right)=\underset{j}{\operatorname{Min}}\left[\operatorname{Max}\left\{\operatorname{Neg}\left(I\left(g_{i}\right)\right), g_{j}\left(a_{i}\right)\right\}\right.
$$

where

$\operatorname{RPC}\left(a_{i}\right)$ is the Risk Priority Code for the failure mode $a_{i}$.

$I\left(g_{j}\right)$ is the importance associated with each criterion $g_{j}$.

$\operatorname{Neg}\left(I\left(g_{j}\right)\right)$ is the negation of the importances assigned to each decisionmaking criterion.

The negation of an s-point ordinal scale is calculated as follows (Yager 1981, 1993):

$$
\operatorname{Neg}\left(L_{i}\right)=L_{z-i+1},
$$

where $L_{i}$ is the $i$ th level of the scale.

(ii) Determination of the failure mode with the maximum risk priority code $\left(a^{*}\right)$

$$
R P C\left(a^{*}\right)=\operatorname{Max}_{a_{i} \neq A}\left\{R P C\left(a_{i}\right)\right\},
$$

where

$A$ is the set of failure modes.

$R P C\left(a_{i}\right) \quad$ is defined on a new 10-point ordinal scale as those values utilized for expressing index evaluations.

If two or more failure modes have the same risk priority code we may obtain a more detailed selection considering the indicator $T\left(a_{i}\right)=\operatorname{Dim} A\left(a_{i}\right)$, where the operator $\operatorname{Dim} A\left(a_{i}\right)$ gives the number of elements contained in the set $A\left(a_{i}\right)$, and $A\left(a_{i}\right)=\left\{g_{j}\left(a_{i}\right) \mid g_{j}\left(a_{i}\right)>R P C\left(a^{*}\right)\right\}$. This term represents a second-step investigation for establishing a measure of the dispersion of criteria, related to a specific failure mode, around the $R P C$ index. It gives an estimation of how many important criteria with high evaluations, compared with the calculated $R P C$, are present in the evaluation of each failure mode.

It is assumed that the importance associated with each evaluation criterion is defined on a 10-point ordinal scale similar to those used for index scales. It is also assumed that the same danger level corresponds to the same ordinal level on the different scales. Table 5 shows the correspondence map between the severity, occurrence and detectability indexes and their related importances. If the four scales do not have the same number of levels the mappings can become more complex. 


\begin{tabular}{lllll}
\hline Level & \multicolumn{1}{c}{$S$ Index } & \multicolumn{1}{c}{$O$ Index } & \multicolumn{1}{c}{$D$ Index } & $I(S, O, D)$ \\
\hline$L_{1}$ & No & Almost never & Almost certain & No \\
$L_{2}$ & Very slight & Remote & Very high & Very low \\
$L_{3}$ & Slight & Very slight & High & Low \\
$L_{4}$ & Minor & Slight & Moderate high & Minor \\
$L_{5}$ & Moderate & Low & Medium & Moderate \\
$L_{6}$ & Significant & Medium & Low & Significant \\
$L_{7}$ & Major & Moderately high & Slight & Major \\
$L_{8}$ & Extreme & High & Very slight & High \\
$L_{9}$ & Serious & Very high & Remote & Very high \\
$L_{10}$ & Hazardous & Almost certain & Almost impossible & Absolute \\
\hline
\end{tabular}

Table 5. Correspondence map between severity, occurrence and detectability indexes and the qualitative scale for the importance associated with each evaluation criterion.

From equation (2) we note that the Min operation selects the smallest of its arguments. If all arguments are high they do not affect the min operation. Consider a criterion that has little importance, it will get an importance rating $L_{k}$ that is low on the scale. When we take the negation of this score we get something high. When we take the Max of the importance criteria with the evaluation $g_{j}\left(a_{i}\right)$ we still get a high score. Thus, we see that low-importance criteria have little effect on the overall 'score'.

It can be shown that the formulation suggested in equation (2) satisfies the properties of Pareto optimality, independence to irrelevant alternatives, positive association of individual scores with overall score and symmetry (Yager 1981, 1993).

An essential feature of this approach is that we have no need to use numeric values and force undue precision on the design team experts.

We note that, in equation (2), we are implicitly assuming a logic to satisfy all characteristics that are important. The term $\operatorname{Max}\left\{\operatorname{Neg}\left(I\left(g_{j}\right)\right), g_{j}\right\}$ indicates a value for a given criterion to the statement 'if the criterion is important, then it has a high score'.

Equation (4) allows the selection of the failure mode with the maximum risk priority code. The rationale of the procedure is to consider the most dangerous failure modes to be those with the highest evaluations on the most important criteria. When two or more failure modes have the same ranking we provide a more detailed selection with the $T\left(a_{i}\right)$ index. $T\left(a_{i}\right)$ defines, for each failure mode, the cardinality of the total number of 'equivalent' risk levels associated with all criteria.

The traditional FMEA is not able to manage situations in which characteristic indexes have different importances. Some authors (Raheja 1991, De Risi 1996) suggest that an appropriate strategy is to analyse all failure modes that are above some specified threshold $R P N$ or above some severity threshold. For instance, a design team might set a policy where all failure modes whose severity is higher than 9 will be analysed in addition to those failure modes whose $R P N$ is above 500 . This approach recognizes the need to differentiate the relative importance of the severity, occurrence and detection indexes, but proposes a rigid scheme in which the severity index is the most important. 
In some particular contexts it might be necessary to change the order of priority among indexes or to change the logic of their analysis. The proposed method overcomes these constraints. It allows a more flexible structure for combining the index importances, and the possibility of defining different technical logics of analysis. If we change, in equation (2), the composition of the operators and the 'tieranking' rule, the design team may build models able to express logics of synthesis different from that proposed. For example, we might define $R P C$ as $R P C\left(a_{i}\right)=\operatorname{Max}_{j}\left\{\operatorname{Min}\left[\left(I\left(g_{j}\right), g_{j}\left(a_{i}\right)\right]\right\}\right.$, which represents a new logic in which the most dangerous failure mode is that with the highest evaluation on the most important criterion.

An application example may better explain the method.

\section{An application example}

Let us consider the example of a design of a cooling fan assembly (see table 1). Let us analyse four different situations.

(a) All characteristic indexes have the same max importance $\left(L_{10}\right)$. This condition is very similar to the 'traditional' FMEA, where all the indexes have the same importance.

$$
I(S)=L_{10} ; \quad I(O)=L_{10} ; \quad I(D)=L_{10} .
$$

Calling $a_{1}, a_{2}, a_{3}$ and $a_{4}$ the four failure modes, the aggregated $R P C$ index calculation is performed as indicated by equation (2).

According to equation (3), the negations of a 10-point ordinal scale are:

$$
\begin{array}{ll}
\operatorname{Neg}\left(L_{1}\right)=L_{10} ; & \operatorname{Neg}\left(L_{6}\right)=L_{5} ; \\
\operatorname{Neg}\left(L_{2}\right)=L_{9} ; & \operatorname{Neg}\left(L_{7}\right)=L_{4} ; \\
\operatorname{Neg}\left(L_{3}\right)=L_{8} ; & \operatorname{Neg}\left(L_{8}\right)=L_{3} ; \\
\operatorname{Neg}\left(L_{4}\right)=L_{7} ; & \operatorname{Neg}\left(L_{9}\right)=L_{2} ; \\
\operatorname{Neg}\left(L_{5}\right)=L_{6} ; & \operatorname{Neg}\left(L_{10}\right)=L_{1} .
\end{array}
$$

Now it is possible to calculate the $R P C$ for the four failure modes (see column six of table 6):

$$
\begin{aligned}
& \operatorname{RPC}\left(a_{1}\right)=\underset{j}{\operatorname{Min}}\left[\operatorname{Max}\left\{\operatorname{Neg}\left(I\left(g_{j}\right)\right), g_{j}\left(a_{1}\right)\right\}\right] \\
& =\operatorname{Min}\left\{\operatorname{Max}\left[\operatorname{Neg}\left(L_{10}\right), L_{5}\right], \operatorname{Max}\left[\operatorname{Neg}\left(L_{10}\right), L_{5}\right],\right. \\
& \left.\operatorname{Max}\left[\operatorname{Neg}\left(L_{10}\right), L_{4}\right]\right\}=\operatorname{Min}\left\{\operatorname{Max}\left[L_{1}, L_{5}\right], \operatorname{Max}\left[L_{1}, L_{5}\right],\right. \\
& \left.\operatorname{Max}\left[L_{1}, L_{4}\right]\right\}=\operatorname{Min}\left\{L_{5}, L_{5}, L_{4}\right\}=L_{4} \\
& R P C\left(a_{2}\right)=\underset{j}{\operatorname{Min}}\left[\operatorname{Max}\left\{\operatorname{Neg}\left(I\left(g_{j}\right)\right), g_{j}\left(a_{2}\right)\right\}\right]=L_{2} \\
& R P C\left(a_{3}\right)=\underset{j}{\operatorname{Min}}\left[\operatorname{Max}\left\{\operatorname{Neg}\left(I\left(g_{j}\right)\right), g_{j}\left(a_{3}\right)\right\}\right]=L_{2} \\
& R P C\left(a_{4}\right)=\operatorname{Min}_{j}\left[\operatorname{Max}\left\{\operatorname{Neg}\left(I\left(g_{j}\right)\right), g_{j}\left(a_{4}\right)\right\}\right]=L_{3} .
\end{aligned}
$$




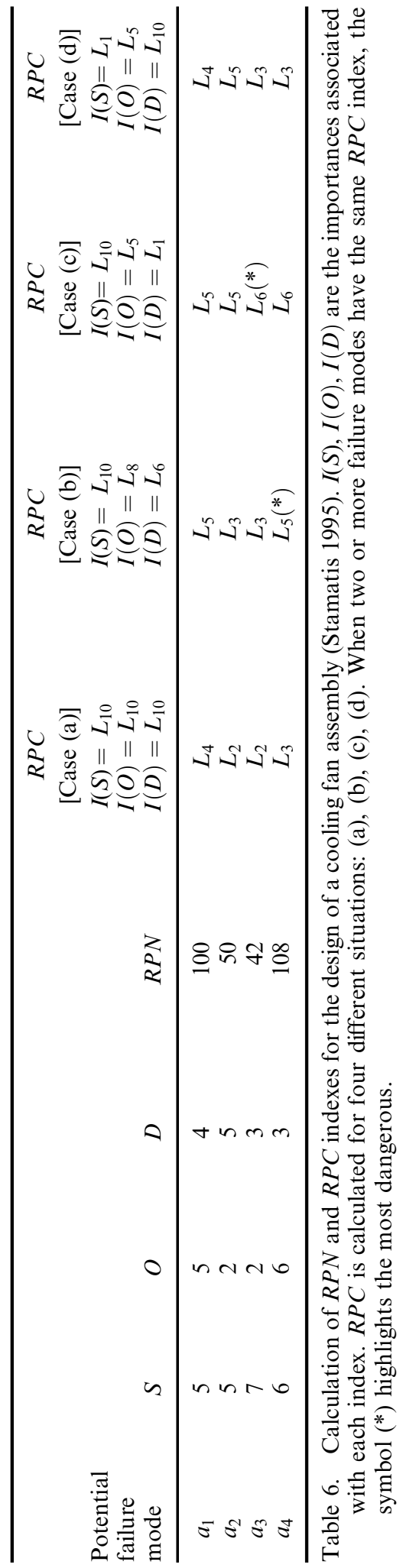


The maximum priority value is:

$$
\begin{aligned}
\operatorname{RPC}\left(a^{*}\right)= & \operatorname{Max}_{a_{i} \in A}\left[\operatorname{RPC}\left(a_{1}\right), \operatorname{RPC}\left(a_{2}\right), \operatorname{RPC}\left(a_{3}\right), \operatorname{RPC}\left(a_{4}\right),\right] \\
= & \operatorname{RPC}\left(a_{1}\right)=L_{4}
\end{aligned}
$$

and therefore the most dangerous failure mode is $a_{1}$.

(b) In some application contexts, it can be useful to define a different level of importance for the three $S, O$ and $D$ indexes. In this case we cannot use the traditional $R P N$ approach.

If $I(S)=L_{10} ; I(O)=L_{8} ; I(D)=L_{6}$.

The $R P C$ for the four failure modes (see column seven of table 6) are:

$$
\begin{aligned}
& R P C\left(a_{1}\right)=\operatorname{Min}_{j}\left[\operatorname{Max}\left\{\operatorname{Neg}\left(i\left(g_{j}\right)\right), g_{j}\left(a_{1}\right)\right\}\right]=L_{5} \\
& R P C\left(a_{2}\right)=\underset{j}{\operatorname{Min}}\left[\operatorname{Max}\left\{\operatorname{Neg}\left(i\left(g_{j}\right)\right), g_{j}\left(a_{2}\right)\right\}\right]=L_{3} \\
& R P C\left(a_{3}\right)=\operatorname{Min}_{j}\left[\operatorname{Max}\left\{\operatorname{Neg}\left(i\left(g_{j}\right)\right), g_{j}\left(a_{3}\right)\right\}\right]=L_{3} \\
& R P C\left(a_{4}\right)=\operatorname{Min}_{j}\left[\operatorname{Max}\left\{\operatorname{Neg}\left(i\left(g_{j}\right)\right), g_{j}\left(a_{4}\right)\right\}\right]=L_{5} .
\end{aligned}
$$

The maximum priority value is:

$$
\begin{aligned}
\operatorname{RPC}\left(a^{*}\right) & =\operatorname{Max}_{a_{i} \in A}\left[\operatorname{RPC}\left(a_{1}\right), \operatorname{RPC}\left(a_{2}\right), \operatorname{RPC}\left(a_{3}\right), \operatorname{RPC}\left(a_{4}\right)\right] \\
& =\operatorname{RPC}\left(a_{1}\right)=\operatorname{RPC}\left(a_{4}\right)=L_{5}
\end{aligned}
$$

In this case the most dangerous failure modes are $a_{1}$ and $a_{4}$.

With the aim of discriminating their relative ranking we calculate the indexes $T\left(a_{1}\right)$ and $T\left(a_{4}\right)$ :

$$
\begin{aligned}
& A\left(a_{1}\right)=\left\{g_{j}\left(a_{1}\right) \mid g_{j}\left(a_{1}\right)>R P C\left(a^{*}\right)\right\}=\left\{g_{j}\left(a_{1}\right) \mid g_{j}\left(a_{1}\right)>L_{5}\right\}=\Phi \\
& \Rightarrow \quad T\left(a_{1}\right)=\operatorname{Dim} A\left(a_{1}\right)=\operatorname{Dim} \Phi=0 \\
& A\left(a_{4}\right)=\left\{g_{j}\left(a_{4}\right) \mid g_{j}\left(a_{4}\right)>\operatorname{RPC}\left(a^{*}\right)\right\}=\left\{g_{j}\left(a_{4}\right) \mid g_{j}\left(a_{4}\right)>L_{5}\right\} \\
& =\left\{g_{1}\left(a_{4}\right), g_{2}\left(a_{4}\right)\right\}=\left\{L_{6}, L_{6}\right\} \\
& \Rightarrow \quad T\left(a_{4}\right)=\operatorname{Dim} A\left(a_{4}\right)=2 \text {. }
\end{aligned}
$$

Since $T\left(a_{4}\right)>T\left(a_{1}\right)$ then $a_{4}$ is the most dangerous failure mode.

(c) If $I(S)=L_{10} ; I(O)=L_{5} ; I(D)=L_{1}$

The $R P C$ for the four failure modes (see column eight of table 6) are:

$$
\begin{aligned}
& R P C\left(a_{1}\right)=\operatorname{Min}_{j}\left[\left\{\operatorname{Max}\left\{\operatorname{Neg}\left(I\left(g_{j}\right)\right), g_{j}\left(a_{1}\right)\right\}\right]=L_{5}\right. \\
& R P C\left(a_{2}\right)=\underset{j}{\operatorname{Min}}\left[\left\{\operatorname{Max}\left\{\operatorname{Neg}\left(I\left(g_{j}\right)\right), g_{j}\left(a_{2}\right)\right\}\right]=L_{5}\right. \\
& R P C\left(a_{3}\right)=\operatorname{Min}_{j}\left[\left\{\operatorname{Max}\left\{\operatorname{Neg}\left(I\left(g_{j}\right)\right), g_{j}\left(a_{3}\right)\right\}\right]=L_{6}\right. \\
& R P C\left(a_{4}\right)=\operatorname{Min}_{j}\left[\left\{\operatorname{Max}\left\{\operatorname{Neg}\left(I\left(g_{j}\right)\right), g_{j}\left(a_{4}\right)\right\}\right]=L_{6} .\right.
\end{aligned}
$$


The maximum priority value is:

$$
\begin{aligned}
\operatorname{RPC}\left(a^{*}\right) & =\operatorname{Max}_{a_{i} \in A}\left[\operatorname{RPC}\left(a_{1}\right), \operatorname{RPC}\left(a_{2}\right), \operatorname{RPC}\left(a_{3}\right), \operatorname{RPC}\left(a_{4}\right),\right] \\
& =\operatorname{RPC}\left(a_{3}\right)=\operatorname{RPC}\left(a_{4}\right)=L_{6}
\end{aligned}
$$

and therefore the most dangerous failure modes are $a_{3}$ and $a_{4}$.

Since $a_{3}$ and $a_{4}$ have the same ranking, we calculate $T\left(a_{3}\right)$ and $T\left(a_{4}\right)$ :

$$
T\left(a_{3}\right)=1 ; \quad T\left(a_{4}\right)=0 .
$$

Being $T\left(a_{3}\right)>T\left(a_{4}\right)$, we conclude that $a_{3}$ is more dangerous than $a_{4}$.

(d) If $I(S)=L_{1} ; I(O)=L_{5} ; I(D)=L_{10}$.

The $R P C$ for the four failure modes (see column nine of table 6) are:

$$
\begin{aligned}
& R P C\left(a_{1}\right)=\operatorname{Min}_{j}\left[\left\{\operatorname{Max}\left\{\operatorname{Neg}\left(I\left(g_{j}\right)\right), g_{j}\left(a_{1}\right)\right\}\right]=L_{4}\right. \\
& R P C\left(a_{2}\right)=\underset{j}{\operatorname{Min}}\left[\left\{\operatorname{Max}\left\{\operatorname{Neg}\left(I\left(g_{j}\right)\right), g_{j}\left(a_{2}\right)\right\}\right]=L_{5}\right. \\
& R P C\left(a_{3}\right)=\operatorname{Min}_{j}\left[\left\{\operatorname{Max}\left\{\operatorname{Neg}\left(I\left(g_{j}\right)\right), g_{j}\left(a_{3}\right)\right\}\right]=L_{3}\right. \\
& R P C\left(a_{4}\right)=\operatorname{Min}_{j}\left[\left\{\operatorname{Max}\left\{\operatorname{Neg}\left(I\left(g_{j}\right)\right), g_{j}\left(a_{4}\right)\right\}\right]=L_{3} .\right.
\end{aligned}
$$

The maximum priority value is:

$$
\begin{aligned}
\operatorname{RPC}\left(a^{*}\right) & =\operatorname{Max}_{a_{i} \in A}\left[\operatorname{RPC}\left(a_{1}\right), \operatorname{RPC}\left(a_{2}\right), \operatorname{RPC}\left(a_{3}\right), \operatorname{RPC}\left(a_{4}\right),\right] \\
& =\operatorname{RPC}\left(a_{2}\right)=L_{5} .
\end{aligned}
$$

The most dangerous failure mode is $a_{2}$.

Table 6 contains a synthesis of the $R P N$ and $R P C$ indexes for the example of table 1, using four sets of importances associated with each index.

Analysing the data contained in table 6 we can observe:

- lowering the importance attached to a particular index (for example, the detectability index), decreases its influence on the selected failure mode;

- the $R P C$ index allows analysis of application cases in which we have a different importance for the three input indexes. This cannot be done by using the $R P N$ index.

- if two or more failure modes have the same $R P C$, it is possible to perform a more detailed selection with the help of the $T\left(a_{i}\right)$ index. In such a way we can discriminate 'tie' situations in which the $R P N$ gives the same result. Let us consider, for example, the two conditions assigning respectively the values $(6$, $1,1)$ and $(2,3,1)$ to $(S, O, D)$ indexes;

- the mapping of failure modes on the $R P C$ scale gives their relative importance only. The absolute value assumed is not important. So, for example, according to table 5 , the level $L_{4}$ means that the corresponding failure mode has a priority lower than $L_{5}$ and higher than $L_{3}$;

- it must be noted that the proposed method allows for a more flexible structure for combining the indexes and defining different technical logics of analysis. 


\section{Conclusions}

This paper introduces and discusses the application of a new method to calculate the risk priority level for the failure mode in FMEA. Data processing is performed by working exclusively on the ordinal features of qualitative scales used to collect information from designers. The method's processing simplicity is comparable with the $R P N$ calculation.

The main novel elements of the proposed method are:

- it does not require any arbitrary and artificial scaling of collected information;

- it is able to deal with situations having different importance levels for the three failure mode component indexes;

- it is able to aggregate design team information, even if they are expressed on ordinal qualitative scales;

- it is easy to computerize.

\section{References}

Bellman, R. E. and Zadeh, L. A., 1970, Decision making in a fuzzy environment. Management Science, 17, 141-164.

Bowles, J. B. and Pelaez, C. E., 1995, Fuzzy logic prioritization of failures in a system failure mode, effects and criticality analysis. Reliability Engineering and System Safety, 50, 203-213.

Bowles, J .B., 1988, The new SAE FMECA standard. Proceedings of the Annual Reliability and Maintainability Symposium, Anaheim, CA, pp. 48-53.

De Risi, P., 1996, Progettare in Qualità, Il Sole 24 Ore Libri, Milano.

Franceschini, F. and Rossetto, S., 1995a, QFD: the problem of comparing technical/engineering design requirements. Research in Engineering Design, 7, 270-278.

Franceschini, F. and Rossetto, S., 1995b, Quality \& innovation: a conceptual model of their interaction. Total Quality Management, 6(3), 221-229.

Franceschini, F. and Rossetto, S., 1997, Design for quality: selecting product's technical features. Quality Engineering, 9(4), 681-688.

Franceschini, F. and Rossetto, S., 1998, On-line service quality control: the 'Qualitometro' method. Quality Engineering, 10(4), 633-643.

Fraser, N. M., 1994, Ordinal preference representations. Theory and Decision, 36(1), 45-67.

Goossens, L. H. and Cooke, R. M., 1997, Applications of some risk assessment techniques: formal expert judgement and accident sequence precursors. Safety Science, 26(1/2), 35-47.

Hatty, M. and Owens, N., 1995, Potential failure modes and effects analysis: a business perspective. Quality Engineering, 7(1), 169-186.

Rajeha, D., 1991, Assurance Technologies (New York: McGraw-Hill).

Stamatis, D. H., 1995, Failure Mode and Effect Analysis (Milwaukee, WI: ASQC Quality Press).

YAGER, R. R., 1981, A new methodology for ordinal multiobjective decisions based on fuzzy sets. Decisions Sciences, 12, 589-600.

YAGER, R. R., 1988, On ordered weighted averaging aggregation operators in multi-criteria decision making. IEEE Transactions on Systems, Man and Cybernetics, 18, 183-190.

YAGER, R. R., 1993, Non-numeric multi-criteria multi-person decision making. Group Decision and Negotiation, 2, 81-93.

Yager, R. R. and Filev, D. P., 1994, Essentials of Fuzzy Modeling and Control (New York: Wiley).

Wirth, R., Berthold, B., Kramer, A. and Peter, G., 1996, Knowledge-based support of system analysis for the analysis of failure modes and effects. Engineering Application of Artificial Intelligence, 9(3), 219-229.

ZADEH, L. A., 1975, The concept of a linguistic variable and its application to approximate reasoning: Part 1. Information Sciences, 8, 199-249. Part 2', Information Sciences, 8, 301357, 1975. Part 3, Information Sciences, 9, 43-80, 1976. 